\title{
Electroactive nanowells for spectrographic fluidic memory
}

\section{Bernardo Cordovez, Demetri Psaltis, David Erickson}

Bernardo Cordovez, Demetri Psaltis, David Erickson, "Electroactive nanowells for spectrographic fluidic memory," Proc. SPIE 6645, Nanoengineering: Fabrication, Properties, Optics, and Devices IV, 66451M (11 September 2007); doi: 10.1117/12.734687

SPIE Event: NanoScience + Engineering, 2007, San Diego, California, United States 


\title{
Electroactive Nanowells for Spectrographic Fluidic Memory
}

\author{
Bernardo Cordovez ${ }^{a}$, Demetri Psaltis ${ }^{b}$ and David Erickson ${ }^{a^{*}}$ \\ ${ }^{a}$ Sibley School of Mechanical and Aerospace Engineering, Cornell University \\ Ithaca, NY 14850 \\ ${ }^{\mathrm{b}}$ Section de Génie Électrique et Électronique,Ecole Polytechnique Fédérale de Lausanne, \\ Switzerland
}

\begin{abstract}
Current optical storage devices such as DVDs have their read/write capabilities fundamentally restricted by the diffraction limit of light. We present an optofluidic architecture for storing cocktails of colloidal quantum dots in electroactive nanowell structures. One application of these devices is the development of a fluidic memory approach which could enable the generation, reading and erasing of multiple bit information packages on single light diffraction limited data marks by spectral and intensity multiplexing of quantum dot cocktails. Here we focus on the development of the electroactive nanowell trapping architecture. Briefly, we have shown that by applying an electric potential between a top and bottom Indium Tin Oxide (ITO) electrodes, particles ranging from $5 \mu \mathrm{m}$ polystyrene spheres to $5 \mathrm{~nm}$ quantum dots suspended in solution can be attracted, stored and rejected from a targeted well structure by electrokinetic actuation. Nanowells $100 \mathrm{~nm}$ in diameter and $1 \mu \mathrm{m}$ deep were fabricated by depositing silicon and a small oxide thin film on top of an ITO cover slip, patterning the wells on electron beam resist followed by a series of dry etching steps that leave the ITO substrate exposed in the well sites. When the quantum dots are electrokinetically transported to their sensing sites, they are then excited by a UV-blue light, and their discrete fluorescent signal is captured by a fiber spectrometer. Data erasure can be selectively performed by reversing the polarity of the field and ejecting the quantum dots from the nanowell data marks.
\end{abstract}

Keywords: Optofluidics, Electrokinetics, Quantum dots, Spectrophotometry

\section{INTRODUCTION}

Optical data storage media which use surface defects as the memory data marks are approaching physical limits beyond which individual bits may be too small or too difficult to store. While techniques like Blu-ray enable one to probe smaller optical sites and thus increase storage density, resolution in optical devices will always be hampered by the diffraction limit of light. One of the main challenges for increasing optical data storage density has been to enable multiple bit storage on single diffraction limited data marks. Techniques like digital holography ${ }^{1}$ have addressed the static nature of optical surface probing, and store information throughout the entirety of the optical volume of the recording media. This technique however has the intrinsic limitation of non feasible rewriteability, since an entire data page needs to be modified for every bit to be changed. The advent of optofluidics ${ }^{2}$ presents new approaches for dynamic manipulation and reconfigurability of optical properties and devices at length scales both greater than and smaller than the wavelength of light. These attributes make optofluidics ideal for a spectrographic fluidic memory approach.

The advances in multilayer soft lithography ${ }^{3}$ have led to renewed interest in fluidic memory devices. Logical circuits have been built by discrete binary sample storage ${ }^{4}$ (probing presence of dye or not) or Reynolds fluidic flip flops ${ }^{5}$. However, the storage densities achieved (near $50 \mathrm{Bs} / \mathrm{cm} 2$ ) are around 6 orders of magnitude smaller than those of current DVDs. While making the fluidic features smaller will increase storage densities, issues with fluidic transport arise, and most importantly, they are still incapable of competing with their optical counterparts. Micro and nanofluidics however do enable the unique ability of delivering and mixing discrete samples of different components in very precise amounts, the ability for these to be dynamically reconfigured, and provides the opportunity to exploit spectral and intensity multiplexing, in this case of the functional optical memory element.

\footnotetext{
* Corresponding Author: de54(at)cornell.edu; (607) 255-4861; http://nano.mae.cornell.edu
} 
The principle behind spectral encoding involves creating discrete information packets which contain different intensity levels of different photoluminescent colloidal nanocrystals. For example 10 different intensity levels of an individual species provide 10 different codes which can then be increased exponentially by increasing the number of individual species. Under such a scheme the equivalent number of bits in a single information packet, $\mathrm{N}$, is:

$$
N=\log _{2}\left(I^{M}-1\right)
$$

where I is the number of intensity levels and $\mathrm{M}$ is the number of individual species (though not significant in terms of information density the -1 accounts for the fact that the all zero code cannot be distinguished from the background). The use of 10 different intensity levels with 10 individual species would provide $10^{10}-1$ individual codes equivalent to slightly more than 33-bits in a single information packet. As such shrinking down fluidic storage units to the micronscale could yield similar or even greater storage density than conventional DVDs.

Semiconductor quantum dots [10] are ideal candidates as photoluminescent particles for such an application. They exhibit much narrower FWHMs than traditional organic dyes, enabling one to multiplex in the spectral domain with higher fidelity and sensitivity. In addition, the emission spectrum of quantum dots can be tuned by changing their quantum confinement conditions (smaller dots are red shifted). Furthermore, different species of quantum dots can be excited by a single UV-Blue light source, avoiding the nuisance that organic dyes require optical pumping at various wavelengths. The use of a single illumination source also makes data processing easier since it can be easily filtered off during data reading.

Figure 1 displays the conceptual overview of our envisioned Nan fluidic storage device. The spectral code generator was analyzed in a previous study ${ }^{6}$, hence we focus this paper mainly on the transport and trapping of quantum dots in the bottom electro-active platform. Quantum dots cocktails are pneumatically delivered to specific nanowell sites by using a multilayer soft lithography fluidic architecture. Once the nanoparticles are in the vicinity of the nanowell data marks, they are electrokinetically transported to their sensing sites, they are then excited by a UV-blue light, and their discrete fluorescent signal is captured by a fiber spectrometer. Data erasure can be selectively performed by reversing the polarity of the field and ejecting the quantum dots from wells.

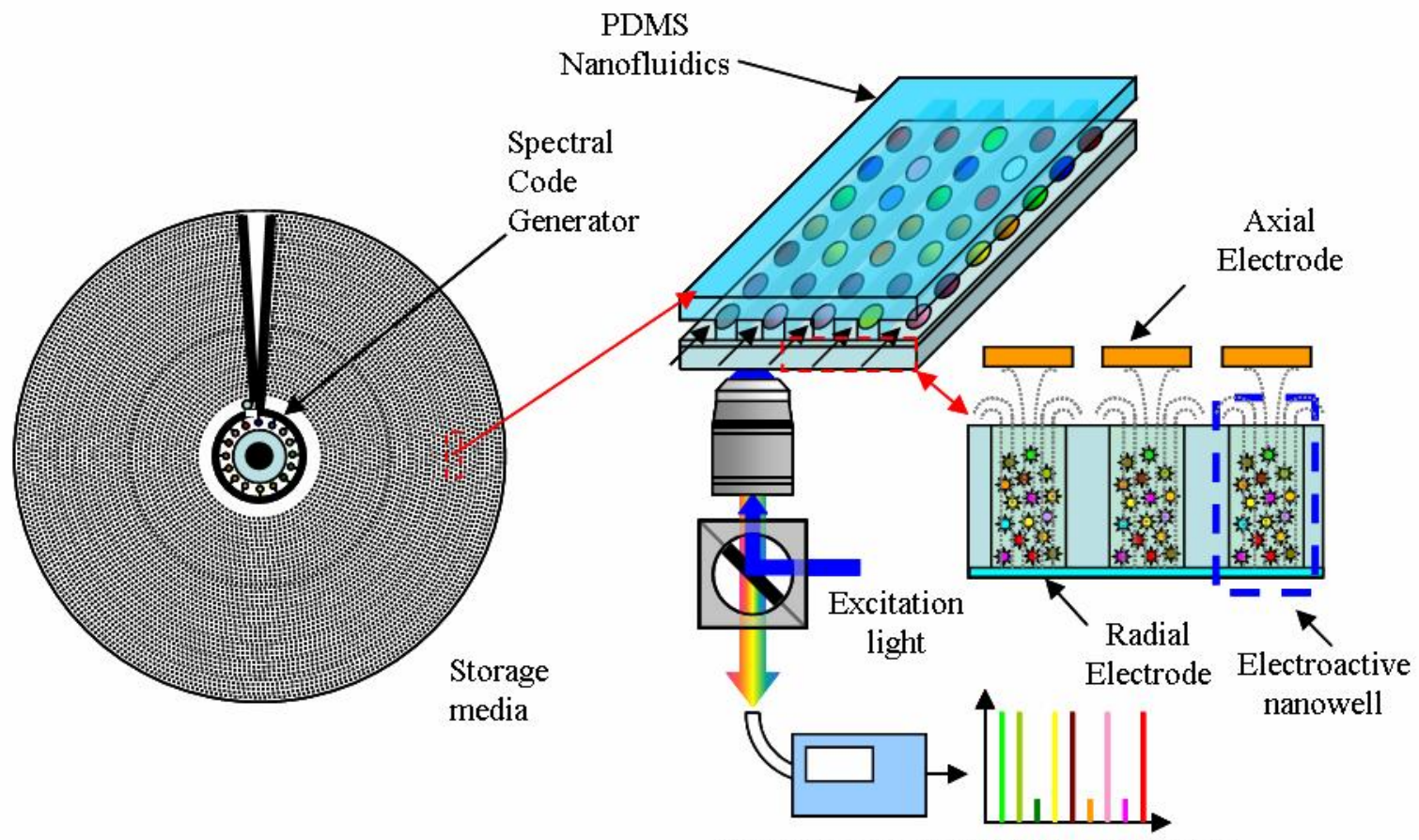

Fiber Spectrometer and Spectral Output

Fig 1. Spectrographic Fluidic Memory Device Layout: Specific Quantum combinations of quantum dot cocktails are generated, transported via pressure driven flow and trapped electrokinetically to the nanowell sites. Data readout is performed by fluorescent excitation and data erasure by reversing the polarity of the electric field. 


\section{THEORY AND PRIOR WORK}

The transport mechanism we use to trap colloidal quantum dots in targeted micro and nano structures is through a combination of electrokinetic effects. Previously, we demonstrated trapping of polystyrene microspheres in a $20 \mu \mathrm{m}$ diameter and $5 \mu \mathrm{m}$ deep electroactive microwell?. Figure 2 below outlines the working mechanism of the electroactive microwell system (a) and demonstrates how the electric field becomes concentrated within the well when the potential is applied as shown (b). Figure 3 shows the attraction, concentration and repulsion of particles into the microwells. In Figures 3 (a) through (d) an attraction voltage is applied and particles (in this case polystyrene spheres) are shown to collect and become concentrated in the well. In (e) and (f) the polarity is reversed and the particles are rejected from the well.

(a)

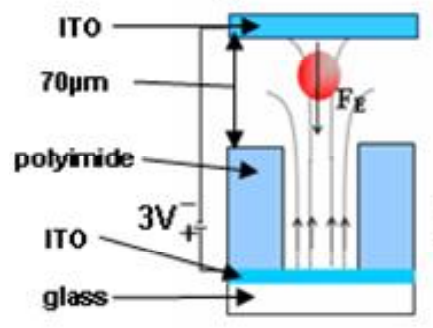

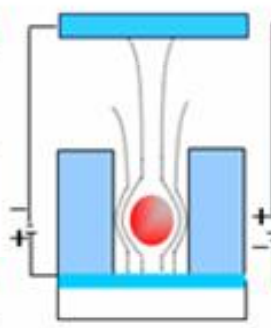

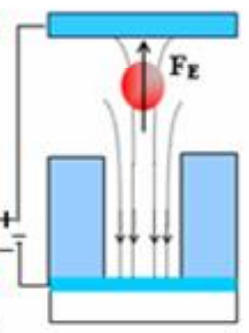

(b)

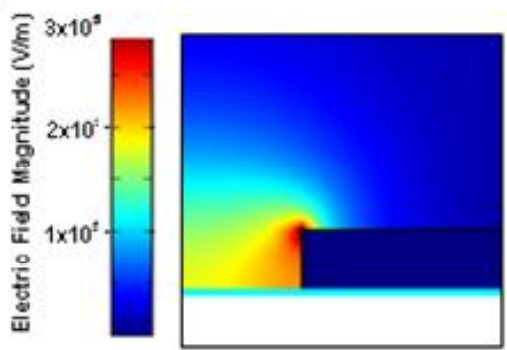

Fig 2. Electroactive Microwells: (a) Particles are driven, stored and repelled from the well depending on the polarity. (b) Axisymmetric finite element simulation displaying strong electric field concentration inside microwell.

(a)
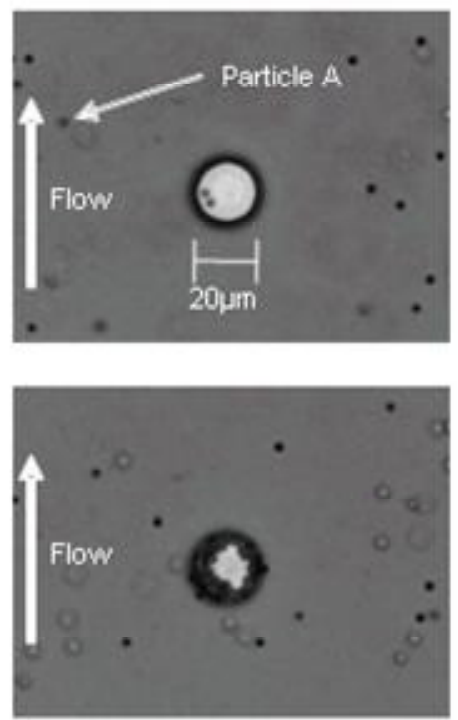

(d) (b)
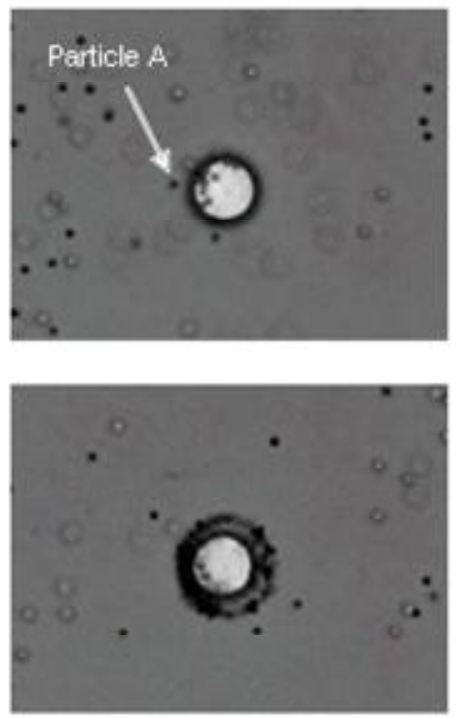

(e) (c)
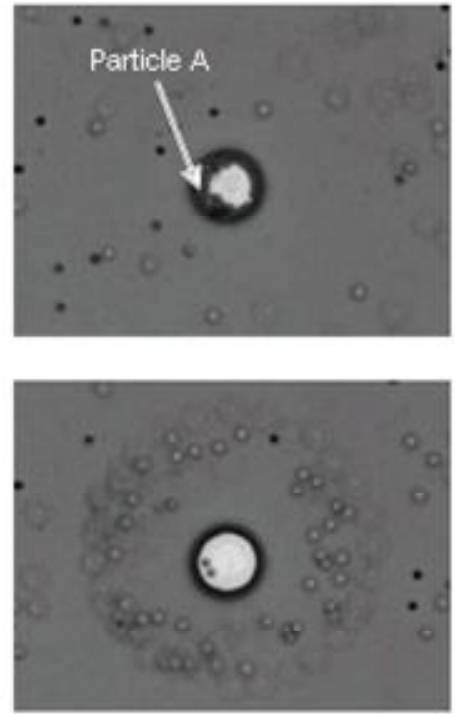

(f)

Fig 3. Time lapse illustrating particle attraction and repulsion of $1.9 \mu \mathrm{m}$ polystyrene beads in a $20 \mu \mathrm{m}$ well. Conditions are a velocity field of $30 \mu \mathrm{m} / \mathrm{s}$ under a 5 Volt potential. (a) Inactive microwell (b-d) Particle attraction begins and beads are drawn into well. (e-f) Particles are rejected abruptly as polarity is switched

A finite element based transport simulation was done to better understand the electrokinetic processes involved in the trapping process. Here we first solved for the Laplace equation for the electric field within the fluidic domain (as shown in Figure 2(b)) and then used the components of the solution to compute the electro-osmotic slip velocity at the surfaces. The low Reynolds number stokes equations are then solved subject to these boundary conditions. The convective transport vectors are then added to the then added to the electrophoretic and dielectrophoretic velocities (computed based from the solution to the Laplace equation) to obtain the total transport velocity. Sample simulation results are shown in Figure 4. 


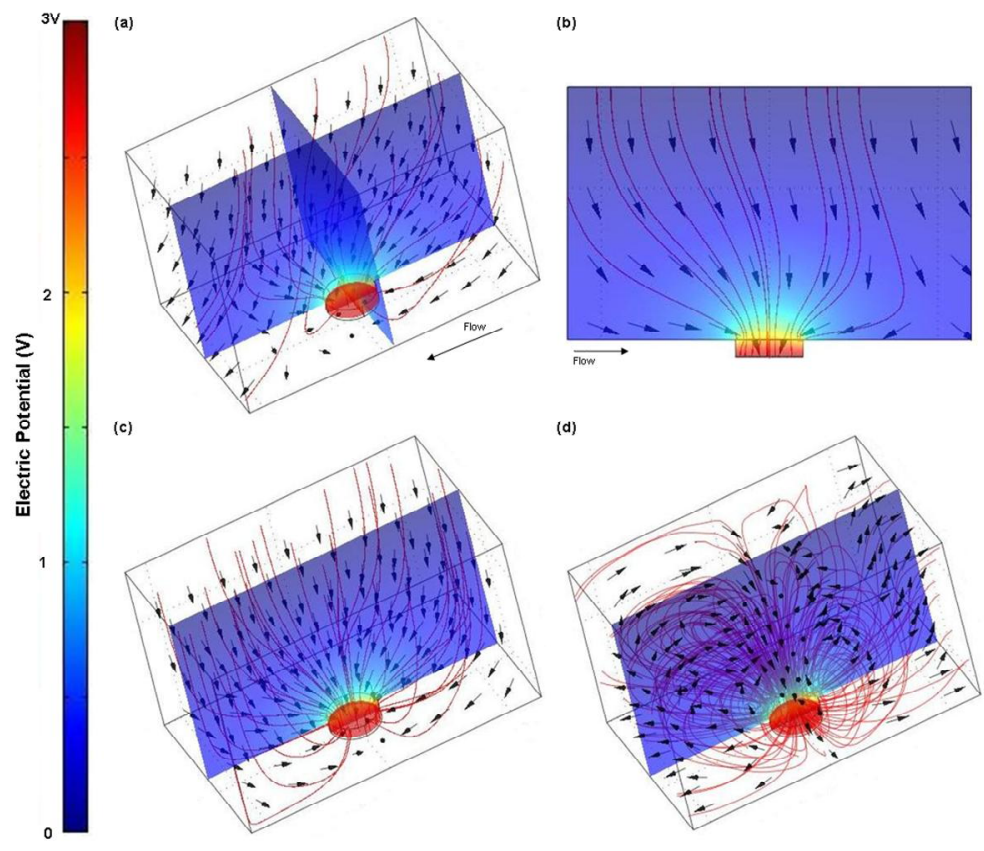

Fig 4. Finite Element Analysis of electrokinetic effects in 20um well: (a) Transport lines illustrate competing effects between electroosmosis, electrophoresis and dielectrophoresis. (b) Transport lines viewed from cross section. (c) Particle trajectories for charged particles with an uncharged surface (d) Recirculation induced by dielectric charging.

\section{EXPERIMENTAL SETUP AND DEVICE FABRICATION}

Microfluidic flow visualization was performed using a 60X oil immersion objective in an inverted microscope, a fluorescence microscopy setup which includes a $100 \mathrm{~W}$ broad band mercury arc lamp, a UV quantum dot filter (Chroma Technology Corp., Rockingham, VT; excitation: bandpass 400nm, emission: longpass 500nm). Streptavidin conjugated quantum dots (Evident Tech. Troy, NY) ranging from $605 \mathrm{~nm}$ to $705 \mathrm{~nm}$ emission wavelengths were used in order to ensure good optical transmission. Images were captured on a black and white CCD camera (Cooke Corp. Romulus, MI) and the spectral measurements were taken using an optical fiber spectrometer (Ocean Optics, Dunedin, FL). High and low pressures used for valving and pressure driven flow respectively were controlled by digital pneumatic manifolds (Fluidigm Corp. South San Francisco, CA). Voltage was supplied by a DC power supply (Hewlett Packard, Palo Alto, CA), and potentials were reversed at will using a custom built polarity changer.

Fabrication of the top fluidic architecture was performed by using a soft lithography poly(dimethylsiloxane) (PDMS) multilayer method following a modified version of the protocols established by Duffy et $\mathrm{al}^{8}$. First, a polyimide mold on a silicon wafer was used as the bottom fluidic layer stamp, followed by a lift off procedure where gold was deposited to generate electrodes sitting conformally orthogonal to the microchannels. A 3mercaptopropyltrimethoxysilane (MPTMS) self assembled monolayer in an isopropyl alcohol (IPA) solution was then soaked on the wafer for 2 hours, a technique established by Lim et al. ${ }^{9}$ in order to transfer the gold onto the PDMS fluidic layer. A thin flexible PDMS membrane was spun on top of the wafer, followed by the introduction of the thick top valving layer. After baking, the PDMS layers were carefully removed from the mold, obtaining the top fluidic architecture shown in Figure 5. Photoactive polyimide (Dow Corning Corp, Midland, MI) was used as the fluidic mold instead of SU-8 (MichroChem Corp. Newton, MA) for the ability to obtain higher sloped sidewalls with positive resist, which eased the pealing process and ensured electrical continuity throughout the gold electrodes. After punching the fluidic access holes, air pressure was delivered form the digital manifolds to the valving control layer, locally collapsing the flow channel and acting as an effective gate valve. 


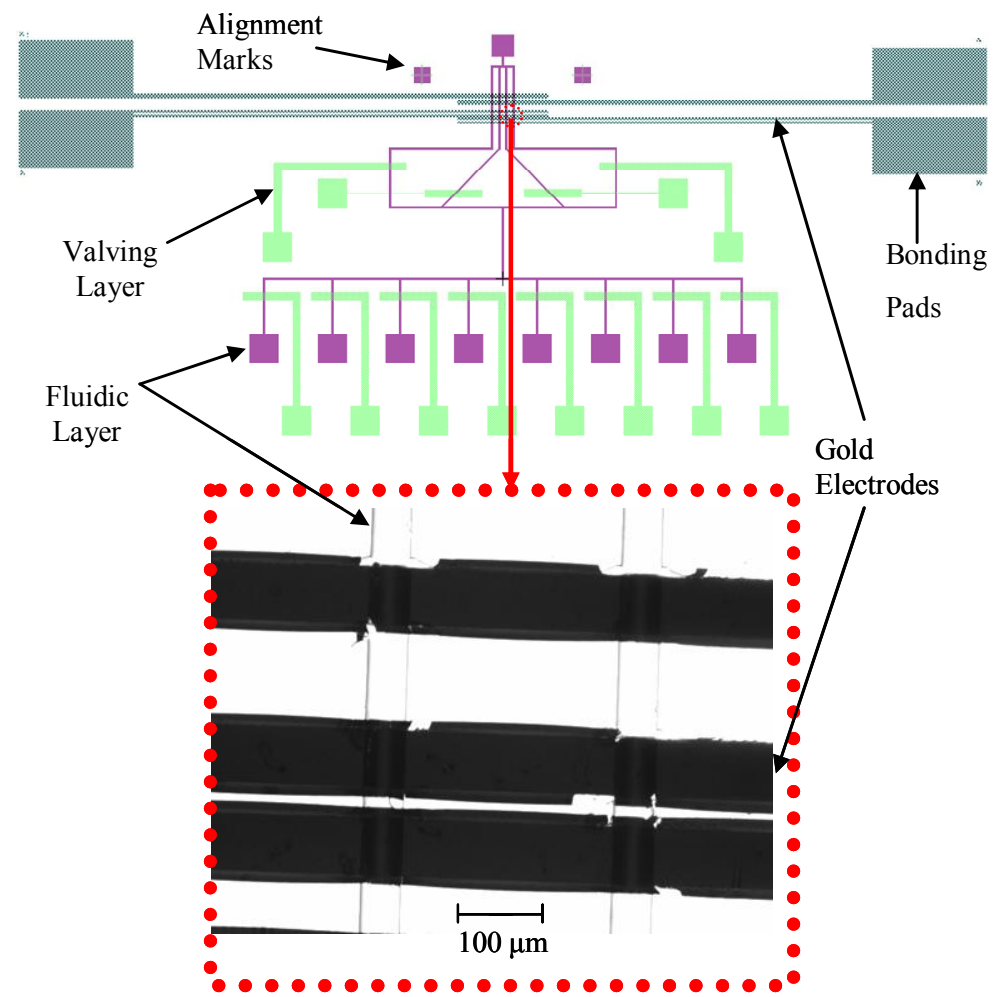

Fig 5. Schematic of quantum dot flow delivery layer. Purple lines represent the thin flow layer and green lines the control layer. The top gold electrodes are placed orthogonal to the flow channels.

To fabricate the electroactive platform, a thin $8-12 \mathrm{ohm}$ indium tin oxide (ITO) coated coverslip (Structure Probe Inc. West Chester, PA) was coated with a thin chrome layer, a thin oxide stop layer, a $600 \mathrm{~nm}$ silicon body and a thin oxide mask. $5 \mu \mathrm{m}$ wells were then patterned on the substrate, followed by a sequence of reactive ion etches and a final wet chrome etch, leaving the ITO layer exposed. ITO was chosen as the bottom transparent conductor in order to enable fluorescent excitation from bellow the substrate, and to function as the bottom conductor for quantum dot attraction during electrokinetic actuation. Figure 6 illustrates our electroactive microwell array device. After fabricating the electroactive microwells (Figure 6a), the channel/electrode junctions of the top multilayer device are aligned and bonded with the electroactive microwells (Figure 6b). Figure 6c shows the entire experimental setup, in which platinum wires are attached to the bonding pads and the bottom ITO electrode through conductive silver epoxy.

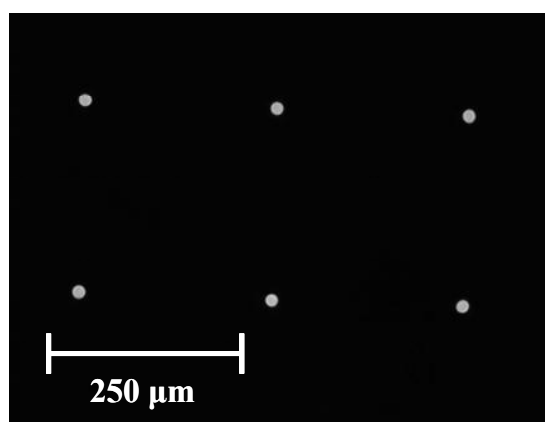

(a)

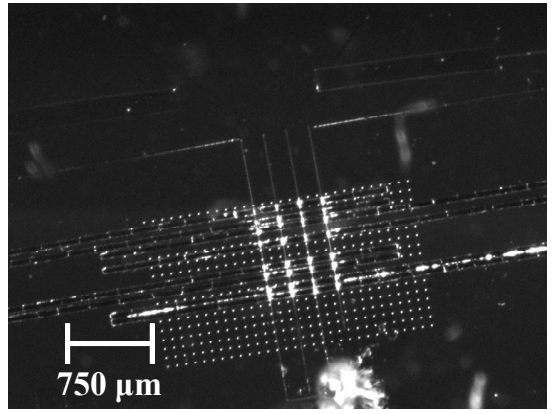

(b)

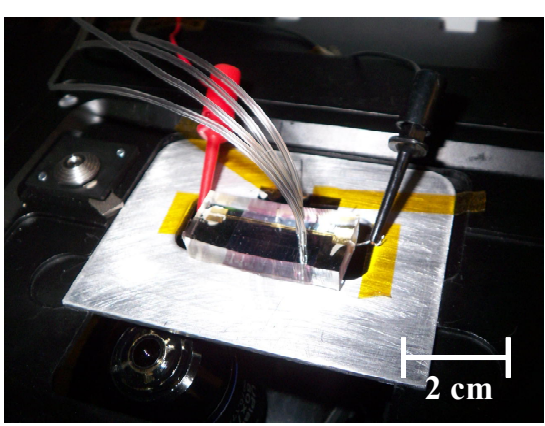

(c)

Fig 6. Schematic of Electroactive Microwell Device: (a) Electroactive microwell array. (b) Multilayer layer flow device with gold electrodes on top of the bottom microwell structure. (c) Full scale experimental setup. 


\section{EXPERIMENTAL RESULTS AND DISCUSSION}

In our first experiment, a $4 \mathrm{nM}$ solution quantum dots with an emission wavelength of $705 \mathrm{~nm}$ was immersed in a $10 \mathrm{mM}$ phosphate buffer solution. Since the colloidal quantum dots are tagged with streptavidin $(\mathrm{pI} \sim 5)$, they support a negative charge in aqueous $\mathrm{pH}$. They were then introduced to the fluidic structure, and can be pumped and valved to a channel with the well of interest. Individual addressability of a single well is possible due to the orthogonality between the top electrode and the channel. Figure 7 presents a time lapse of images displaying the attraction and repulsion of red quantum dots, visible by changes in the fluorescent excitation thru the well. The signal in Figures $7 \mathrm{~b}$ and $7 \mathrm{~d}$ is representative of the background signal of the quantum dots above the well. In our future devices a flushing step will be used to carry away the quantum dots after they are rejected. A 1.1 Volts potential was applied between the top gold electrode and the base ITO electrode. Figure 8 displays the spectral signal obtained from the well site.

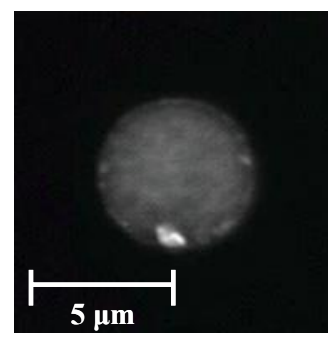

(a)

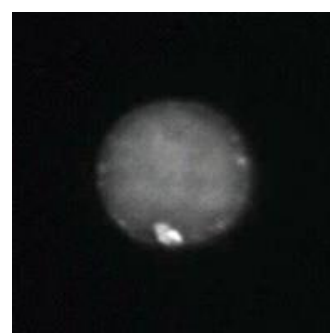

(c)

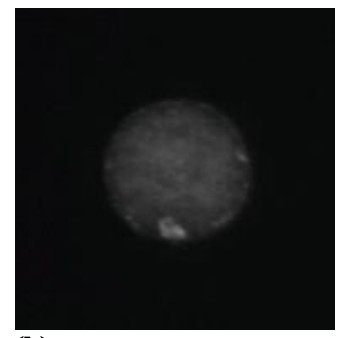

(b)

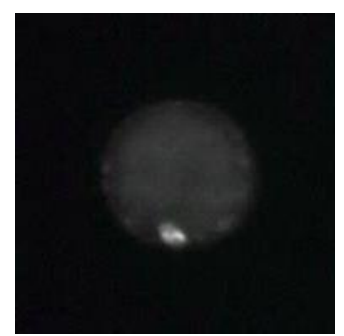

(d)

Fig 7. Time lapse illustrating quantum dot attraction and repulsion in a $5 \mu \mathrm{m}$ well. Conditions are a velocity field of $10 \mu \mathrm{m} / \mathrm{s}$ under a 1.1 Volt potential. (a) Electroactive microwell in attraction mode for 9 seconds. (b) Quantum dot rejection. (c) Polarity is reversed and attraction resumes after 11 seconds. (d) Quantum dots are repulsed again from the well site.

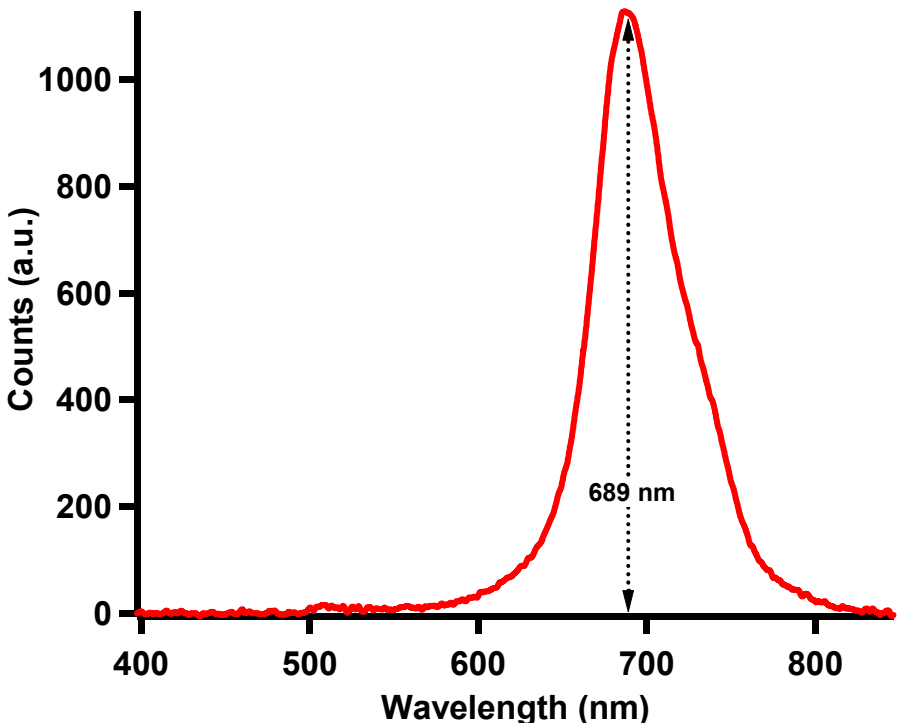

Fig 8. Spectrum of $705 \mathrm{~nm}$ quantum dots thru an electroactive microwell. A reference dark spectrum taken in the electroactive structure but outside the microwell was subtracted from the well readout to obtain this signal. 
Attraction and repulsion switching times were instantaneous at this potential (approximately a few milliseconds). Higher voltages enable faster switching speeds, however one risks charring of the ITO electrode, making it more difficult to detect changes in intensity, as well as generating undesired electrolysis or dielectric breakdown of the thin oxide layer.

To demonstrate the electrokinetic trapping, reading and repulsion of two different species of quantum dots at different intensities, a $3 \mathrm{nM}$ solution of $705 \mathrm{~nm}$ quantum dots was mixed with a $6 \mathrm{nM}$ solution of $605 \mathrm{~nm}$ quantum dots and delivered to a microwell data mark. Figure 9 displays a time lapse of images showing attraction and repulsion of this quantum dots mixture.

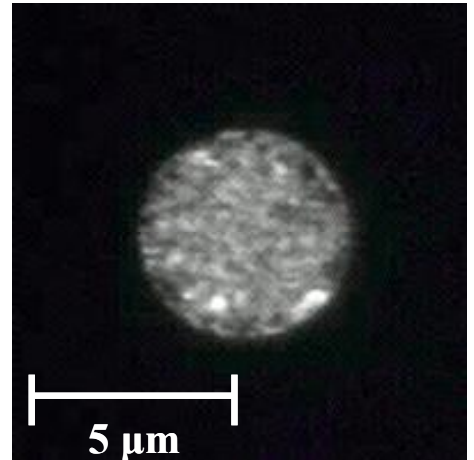

(a)

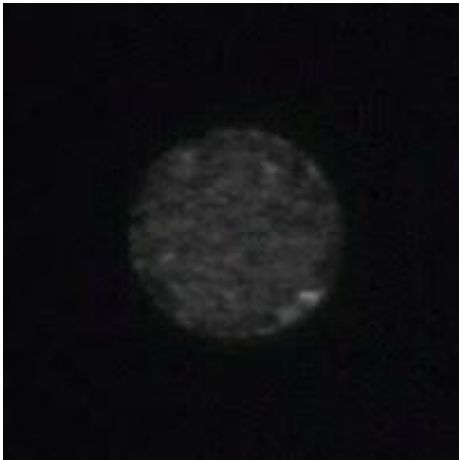

(b)

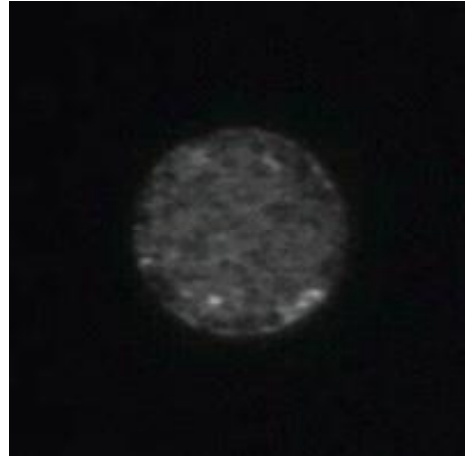

(c)

Fig 9. Time lapse illustrating quantum dot attraction and repulsion in a $5 \mu \mathrm{m}$ well. Conditions are a velocity field of $10 \mu \mathrm{m} / \mathrm{s}$ under a 1.1 Volt potential. (a) Electroactive microwell in attraction mode for 11 seconds. (b) Quantum dot rejection. (c) Polarity is reversed again and attraction resumes after 17 seconds.

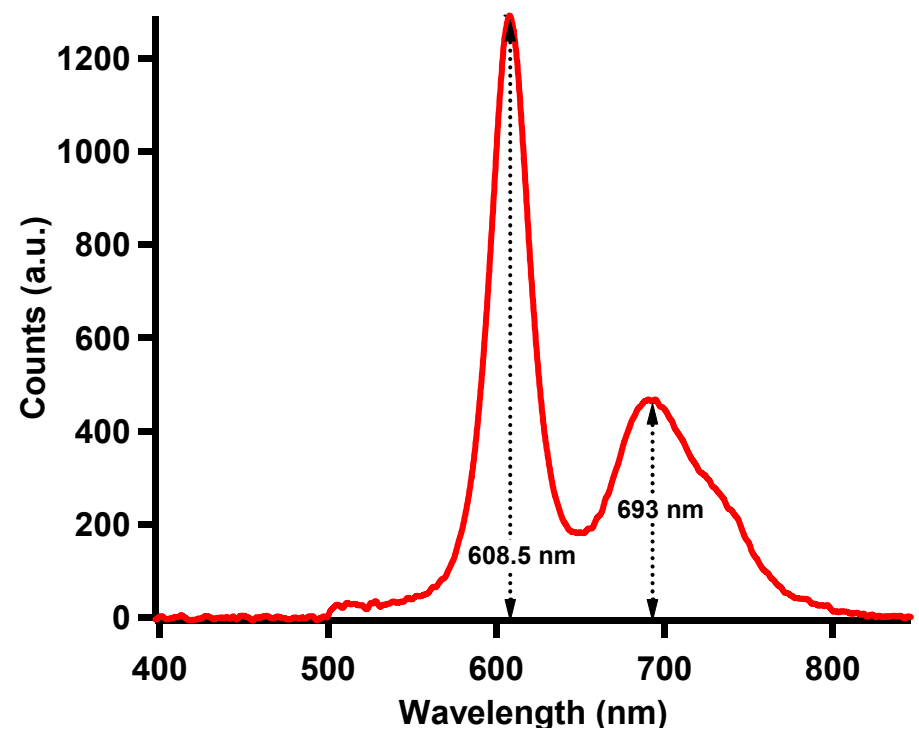

Fig 10. Spectrum of $6 \mathrm{nM}$ solution of $605 \mathrm{~nm}$ quantum dots and $3 \mathrm{nM} 705 \mathrm{~nm}$ quantum dots thru an electroactive microwell.

Even though the contrast between Figures $9 b$ and $9 c$ is very significant and enough to perform a successful spectrographic readout, there is a loss in intensity when comparing the first and second attraction mode states. Possible causes are a gradual charring of the ITO electrode; however a convective flow induced by the UV light source seems more likely. As the regions in which light source is localized (the wells) become hotter, a thermal induced convective flow is generated which makes the flow and particles travel from hotter to colder regions, resulting in a drop in intensity as the experiment continues. 


\section{CONCLUDING REMARKS AND FUTURE EXPERIMENTS}

In summary, we have established an electroactive microwell trapping architecture which serves to capture, store and actively repel quantum dots from confined geometries using electrokinetic actuation. The concentration of the electric field within the well enables high trapping potentials which are suitable for trapping the colloidal nanoparticles. Our current efforts are focused in performing similar experiments in nanowells $100 \mathrm{~nm}$ in diameter, as shown in Figure 11. A reduction of the well size to these dimensions would enable trapping densities roughly 33 times higher than that available today by Blu-Ray. Current simulations to better understand the flow in the nanoscale are also currently been explored.

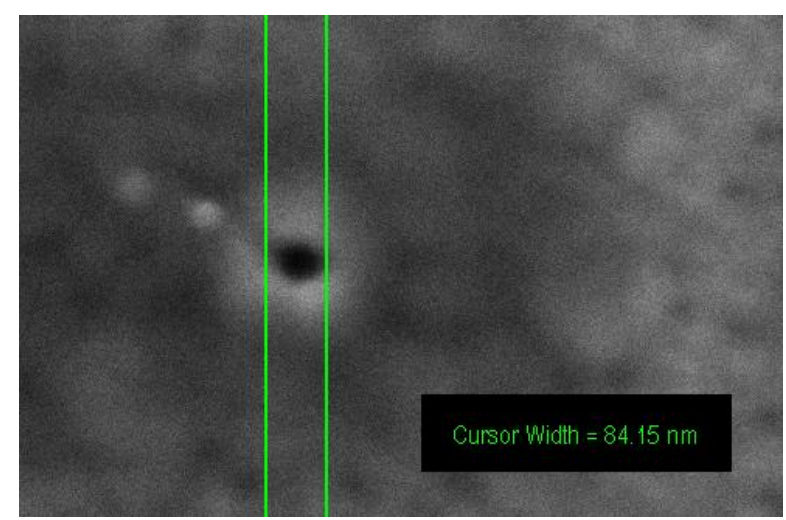

Fig 11. $85 \mathrm{~nm}$ well fabricated by electron beam lithography

\section{ACKNOWLEDGMENTS}

This work is supported by National Science Foundation through the Sensors and Sensor Networks program under grant NSF/CTS 0529045.

\section{REFERENCES}

1. Psaltis, D. and Mok, F., "Holographic Memories,” Sci. Amer. 273, No. 5, 70, 1995.

2. Psaltis, D., Quake, S., Yang, C., "Developing optofluidic technology through the fusion of microfluidics and optics" Nature 442, pp. 381-386, 2006

3. Unger, M., Chou, H., Thorsen, T., Scherer, A., Quake, S., "Monolithic microfabricated valves and pumps by multilayer soft lithography" Science, 288, pp. 113-116, 2000.

4. Thorsen, T., Maerkl, S.J., Quake, S.R., "Microfluidic Large Scale Integration" Science, 298, pp. 580-584, 2002

5. Groisman, A., Enzelberger, M., Quake, S., 2003, "Microfluidic memory and control devices" Science, 300, pp. 955958,2003

6. Erickson, D., Li, B., Adleman J.R., Vyawahare, S., Quake' S., Psaltis, D. "Spectrographic Fluidic Memory" Proceedings of ICMM2005: 3rd International Conference on Microchannels and Minichannels June, Toronto, CA, 2005

7. Cordovez, B., Psaltis, D., Erickson D., "Trapping and Storage of Particles in Electroactive Microwells." Applied Physics Letters, 90, 024102, 2007.

8. Duffy, D., McDonald, J., Schueller, O., Whitesides, G., "Rapid prototyping of microfluidic systems in poly(dimethylsiloxane)" Anal. Chem. 70 pp. 4974-4984., 1998.

9. Lim, K.S., Chang, W., , Yoon-Mo Koo, Y., Bashir, R., "Reliable fabrication method of transferable micron scale metal pattern for poly(dimethylsiloxane) metallization" Lab Chip, 6, pp. 578-580, 2006. 